\title{
Oximetría, Frecuencia Cardíaca, Apnea y Actividad de los Músculos Masticatorios en Sujetos con BS
}

\author{
Oximetry, Heart Rate, Apnea and Mastication \\ Muscle Activity in Subjects with Sleep Bruxism
}

\author{
Olga Patricia López Soto'; Lina María López Soto1; Yéssica Paola López Echeverry'; \\ Cynthia Alejandra Garibay Parra ${ }^{2} \&$ Betsabe Carmona Rocha ${ }^{2}$
}

LÓPEZ-S. O. P.; LÓPEZ-S. L. M.; LÓPEZ- E. Y. P.; GARIBAY-P. C. A. \& CARMONA, R. B. Oximetría, frecuencia cardíaca, apnea y actividad de los músculos masticatorios en sujetos con bruxismo del sueño. Int. J. Odontostomat., 14(1): 42-47, 2020.

RESUMEN: El objectivo de este estudio fue describir los valores promedio de oximetría, frecuencia cardíaca y eventos de apnea durante la noche y la actividad de los músculos maseteros y temporales en un grupo de adultos jóvenes con BS. Estudio observacional descriptivo de serie de casos que consideró una muestra 20 jóvenes entre 21 y 25 años diagnosticados con BS mediante polisomnografía. Se describieron las condiciones de actividad de los músculos maseteros y temporales mediante electromiografía y los valores e los signos vitales mediante polisomnografía. El análisis estadístico fue de tipo descriptivo, se aplicaron medidas de tendencia central, frecuencias absolutas y relativas. El promedio de la actividad muscular durante la masticación de los maseteros fue (D: 86) (I: 520). El promedio de la actividad muscular durante la masticación de los temporales fue (D: 340 ) (I: 510$)$. El promedio en los episodios de hipopnea fue de $(28,9)$. El promedio de oximetría fue (NMOR: 93,5) (MOR: 93,8). El promedio de la tasa cardiaca fue (NMOR: 65,4) (MOR: 66,8) latidos/minuto. El promedio de eventos de bruxismo fue (NREM: 135,4) (REM: 120,2).y el índice (eventos BS/hora) $(40,60)$. Las características de las musculares masticatorias y los signos vitales durante el sueño de los sujetos con BS pueden ayudar al entendimiento y a la toma de decisiones en el tratamiento.

PALABRAS CLAVE: bruxismo (MeSH), bruxismo del sueño (MeSH), masticación (MeSH), polisomnografía (MeSH), oclusión dental (MeSH), contracción muscular (MeSH).

\section{INTRODUCCIÓN}

El bruxismo del sueño (BS) es una actividad muscular masticatoria que ocurre durante el sueño, y que se caracteriza por ser rítmica (fásica) o tónica (Lobbezoo et al., 2013). El BS se considera una actividad multi-sistémica compleja de etiología multifactorial aún por determinar (Klasser et al., 2015). EI BS se clasifica según su etiología en bruxismo primario o idiopático, que no tiene una causa identificable ni ningún problema socio-psicológico o médico asociado; y secundario, que está relacionado con una afección socio-psicológica o médica (p. ej., trastorno del movimiento o del sueño que incluye un trastorno periódico del movimiento de las extremidades y trastornos del movimiento rítmico, como golpes de cabeza y alteraciones respiratorias asociadas a trastor- nos del sueño debido a la resistencia de las vías respiratorias superiores o apnea-hipopnea (Saito et al., 2013) El BS puede tener consecuencias oro-dentales secundarias como fracturas dentales, alteración en la función oclusal y en la articulación temporomandibular, dolor o limitación de los movimientos de los músculos oro-faciales y cefaleas temporales (Choi et al., 2002).

Es imperativo que como mínimo, los estudios sobre el bruxismo del sueño se centren en la masticación y actividad muscular. Existe controversia sobre si el bruxismo debería ser considerado una alteración, Raphael et al. (2016) señalaron que los niveles más altos de actividad muscular masticatoria

\footnotetext{
1 Universidad Autónoma de Manizales, Colombia.

${ }^{2}$ Universidad Autónoma de Ciudad Juárez, México.
} 
aumentaban el riesgo de consecuencias negativas en la salud oral (p. ej., dolor muscular masticatorio severo o dolor en la articulación temporo-mandibular, desgaste mecánico extremo de los dientes, o complicaciones prostodónticas) (Manfredini et al., 2014). Dependiendo de la intensidad, frecuencia y duración, el BS podría afectar el sistema estomatognático (Carlsson et al., 2003), incluso se ha reportado en la literatura efectos perjudiciales en la dentición cuando el BS se ha iniciado tempranamente (Restrepo et al., 2001).

El bruxismo debe considerarse un factor de riesgo en lugar de una alteración o trastorno en los individuos sanos. Los factores psicológicos y el estrés han sido asociados a la etiología del bruxismo del sueño. Estudios anteriores han demostrado que la actividad muscular masticatoria durante el sueño y las mialgias diurnas aumentan durante los periodos estresantes (Clark et al., 1979). Raphael et al. (2013) demostraron que los pacientes con BS, dolor muscular facial diurno y de ATM, tenían durante el sueño un aumento en el esfuerzo respiratorio relacionado con micro-despertares y sueño fragmentado. Estos despertares podrían, según los autores, estar relacionados con un aumento en el tono muscular no específico, por lo que se podría pensar en una actividad muscular masticatoria aumentada que se mantendría aún fuera de los periodos de bruxismo.

La masticación es un proceso complejo caracterizado por el desmenuzamiento y ruptura del alimento en pequeñas partículas para facilitar la digestión (Toro et al., 2006). Kobayashi et al. (2012), reportaron mejor desmenuzamiento de los alimentos en un grupo control cuando se comparaba con sujetos con BS, éstos además, tenían una preferencia por una masticación unilateral, que se presentaba con menor amplitud, con predominancia de movimientos verticales mandibulares y con masticación unilateral.

Los patrones de contracción de los músculos masticatorios han sido estudiados por EMG (Kerstein, 2004) que es considerado un método eficaz para controlar los cambios en la actividad muscular masticatoria. El bruxismo podría aumentar la actividad y el volumen de los músculos masticatorios, ocasionando mayor fuerza al morder. Nishigawa et al. (2001) encontraron que la fuerza de mordida nocturna durante el bruxismo puede exceder la máxima fuerza voluntaria durante el día, ya que los mecanismos de protección del sistema nervioso no funcionan en la noche.
La hipótesis más reciente sobre la etiología del bruxismo del sueño se apoya en los roles de los sistemas nervioso central y autónomo que determinan la génesis de la actividad oromandibular durante el sueño. Los estudios han demostrado que la actividad masticatoria de los episodios de BS se produce durante la activación transitoria asociada con la actividad cerebral y cardíaca, como lo demuestra un rápido aumento de la frecuencia cardiaca al bruxar durante el sueño (taquicardia) (Lavigne et al., 2011). El sueño se divide en no REM sueño ligero (etapas 1 y 2) y sueño profundo (etapas 3 y 4) (Carra et al., 2011). La polisomnografía (PSG) es el estudio de los trastornos del sueño que se basa en el registro de eventos fisiológicos durante toda una noche de sueño. Este sistema incluye electroencefalografía, electro-oculografía y electromiografía de los músculos submental / suprahioideo, tibial anterior, mentalis, masetero y temporal, a través de los cuales se pueden identificar los signos del bruxismo del sueño (Trindade \& Rodriguez, 2014). Lavigne et al. (2001) publicaron los resultados de un estudio de "cut off" para determinar cuándo un sujeto podría diagnosticarse como bruxómano del sueño, proponiendo que un promedio de 25 o más estallidos de bruxismo por hora era el valor guía para este diagnóstico.

Todas estas teorías sobre la fisiología del BS, justifican que se describa en particular el comportamiento fisiológico al dormir de los sujetos con BS, como un aporte al entendimiento integral de lo que ocurre en éstos sujetos a partir de resultados polisomnográficos y electro-miográficos que ayuden en el diagnóstico del BS y en la toma de decisiones en su tratamiento.

En este trabajo el objetivo fue describir los valores promedio de oximetría, frecuencia cardíaca y eventos de apnea/hiponea durante la noche y la actividad de los músculos maseteros y temporales en un grupo de adultos jóvenes con BS.

\section{MATERIAL Y MÉTODO}

Este estudio observacional descriptivo de corte transversal, de serie de casos que consideró 20 jóvenes adultos entre 23 y 25 años que fueron diagnosticados en un macro-proyecto con BS. Se consideraron las siguientes condiciones de exclusión: tratamiento dental en curso, más de cuatro reconstrucciones prostodónticas conservativas de las coronas, más de 
dos zonas desdentadas no funcionales, excluyendo los terceros molares, presencia de prótesis dentales removibles o restauraciones protésicas extensas y presencia de maloclusiones severas.

Se solicitó autorización a la dirección del laboratorio del sueño de la UAM, para el uso de la base de datos de los registros polisomnográficos con fines investigativos y al comité de bioética de la UAM para el uso de los registros polisomnográficos del laboratorio del sueño de esta entidad cumpliendo lo exigido por la norma 8430 que rige la investigación en salud. No se utilizaron los nombres de los pacientes por lo tanto se garantizó el mantenimiento de la privacidad en los datos

La actividad de los músculos masticatorios se determinó por electromiografía de superficie (sEMG) El registro sEMG se realizó con el electrofisiógrafo y software Sierra ${ }^{\circledR}$ Wave ${ }^{\circledR}$ de Cadwell $\AA$. Los parámetros de adquisición se ajustaron a una frecuencia de muestreo de $76,8 \mathrm{kHz}$, un filtro pasa bandas de 10 a $10 \mathrm{kHz}$, y $200 \mu \mathrm{V}$ de ganancia. Para la adquisición y almacenamiento de los registros se seleccionaron el protocolo sEMG. Se utilizaron electrodos dedisco Cadwell $® 302139-200$ de acero inoxidable y $10 \mathrm{~mm}$ de diámetro.

Los electrodos se posicionaron de la siguiente manera: Para el masetero se solicitó al paciente cierre dentario con máxima fuerza, se palpó el músculo masetero, un electrodo activo se ubicó en el punto motor y un electrodo de referencia a $1 \mathrm{~cm}$ por debajo del lóbulo de la oreja. Para el temporal se solicitó al paciente apretar en máxima intercuspidación dental y se palpó la contracción del vientre anterior del temporal.

La PS consideró una electro-oculografía, un electromiograma, una medida de flujo de aire, la medida del esfuerzo respiratorio, la medida de la presencia de ronquido, el monitoreo de la función cardíaca, la medida de la oxigenación y el monitoreo de la posición del cuerpo. La Electroencefalografía tomó la actividad eléctrica superficial del cerebro y permitió identificar las etapas del sueño y del insomnio. Los sensores de la PS fueron fijados en el paciente de una manera no-invasiva, con el uso de una cinta adhesiva (fixomull). La aplicación de los electrodos y sensores se consideró la parte más crítica del examen por lo que se cumplió el protocolo de re-chequeo de cada uno de los puntos que fue monitoreado, dicho re-chequeo fue consignado en la bitácora del examen. La polisomnografía fue analizada después del registro de las etapas del sueño por parte de una fisiatra entrenada y calibrada en investigación de alteraciones del sueño. La información se recolectó a partir de la herramienta de análisis automático incorporado en el software del polisomnógrafo Cadwell Easy III, versión actualizada en 2016. Los datos fueron registrados en el programa estadístico SPSS versión 23.

La actividad muscular fue valorada según la cualificación de la intensidad de la actividad muscular en microvoltios propuesta por Basmajian1: (Insignificante: 0-50; ligera 51-150; Moderada 151-300; marcada 301-500; mayor a 501 muy marcada. El índice de asimetría se consideró compatible con una función normal hasta un valor de $18 \%$ siguiendo las indicaciones de Ferrario et al. (1993).

Las variables cuantitativas se analizaron con medidas de tendencia central y medidas de dispersión como la varianza, la desviación estándar y el coeficiente de variación. Las variables categóricas se analizaron mediante frecuencias absolutas y relativas.

\section{RESULTADOS}

Según la prevalencia del BS, el grupo de adultos jóvenes, incluyó 22 sujetos entre 18 y 25 años de los cuales 5 eran hombres ( $25 \%$ ) y 15 mujeres ( $75 \%$ ) con una edad promedio (DE) de 22,1 años.

\section{Resultados de la actividad muscular en sujetos con}

BS. La actividad durante la masticación del masetero derecho fue ligera y del masetero izquierdo muy marcada. No se encontró asimetría. La actividad durante la masticación del temporal derecho fue marcada y la del temporal izquierdo muy marcada. No se encontró asimetría. (Tabla I).

Resultados de los registros polisomnográficos en sujetos con BS. Los episodios de hipopnea en los pacientes adultos jóvenes con bruxismo del sueño fueron de 28,9 en promedio. El promedio de la frecuencia cardíaca se encontró dentro de los valores normales (60-100 latidos/min). Se observó un promedio alto del conteo del movimiento periódico de las extremidades. Se presentó un promedio 40,60 eventos de bruxismo por hora. Hay un alto número promedio de eventos de bruxismo en REM $(120,2)$ (Tabla III). 
LÓPEZ-S. O. P.; LÓPEZ-S. L. M.; LÓPEZ- E. Y. P.; GARIBAY-P. C. A. \& CARMONA, R. B. Oximetría, frecuencia cardíaca, apnea y actividad de los músculos masticatorios en sujetos con bruxismo del sueño. Int. J. Odontostomat., 14(1): 42-47, 2020.

Tabla I. Promedio de actividad masticatoria en microvoltios y simetría de maseteros y temporales en jóvenes con BS según electromiografía.

\begin{tabular}{llll}
\hline & X & IC 95 \% & Cualificación \\
\hline $\begin{array}{l}\text { Actividad masticatoria masetero } \\
\text { Derecho }\end{array}$ & 86 & $(-30-200)$ & Ligera \\
Izquierdo & 520 & $(-500-1540)$ & $\begin{array}{l}\text { Muy marcada } \\
\text { Asimetría en masticación }\end{array}$ \\
$\begin{array}{l}\text { Actividad masticatoria temporal } \\
\text { Derecho }\end{array}$ & $3 \%$ & $(-17 \%-23)$ & No hay asimetría \\
Izquierdo & 340 & $(-300-980)$ & Marcada \\
Asimetría en masticación & 510 & $(-500-151)$ & Muy marcada \\
\hline
\end{tabular}

X: Promedio (D.E): Desviación estándar (IC $95 \%$ ): Intervalo de confianza 95 \%

Tabla II. Episodios de apnea, oximetría, tasa cardíaca y eventos de bruxismo en 20 adultos jóvenes con BS.

\begin{tabular}{lll}
\hline & $\mathrm{X}$ & $\mathrm{D} . \mathrm{E}$. \\
\hline Episodios apnea central & 0,6 & 1,3 \\
Episodios apnea obstructiva & 1,4 & 3,8 \\
Episodios de apnea mixta & 0,7 & 1,5 \\
Episodios de hipopnea & 28,9 & 33,2 \\
Oximetría NMOR & 93,5 & 1,4 \\
Oximetría en MOR & 93,8 & 1,3 \\
Tasa cardíaca ( latidos/min)en NMOR & 65,4 & 6,6 \\
Tasa cardiaca (latidos/min) en MOR & 66,8 & 7,1 \\
Conteo de movimientos periódicos de las extremidades totales & 84,2 & 45,8 \\
Eventos de bruxismo & 260,5 & 119,0 \\
Eventos de Bruxismo NREM & 135,2 & 102,1 \\
& 135,4 & 77,8 \\
Eventos de Bruxismo REM & 120,2 & 90,2 \\
Índice de Bruxismo & 40,60 & 18,9
\end{tabular}

X promedio D.E. Desviación Estándar

\section{DISCUSIÓN}

En el presente trabajo investigativo la actividad muscular del músculo masetero derecho fue ligera y del izquierdo muy marcada, se registró además una actividad masticatoria del temporal derecho marcada y del izquierdo muy marcada. Las contracciones musculares frecuentes durante el periodo del sueño podrían afectar negativamente la correcta función de los músculos masticatorios. En pacientes con bruxismo crónico se ha observado una marcada hipertrofia maseterina, debido a que el bruxismo conlleva a una excesiva actividad muscular. Onozuka et al. (2002) afirmaron que aplicación de fuerzas musculares repetitivas sobre los dientes podía ocasionar desgaste dental, dolor oro-facial, hiperactividad e hipertrofia muscular, especialmente del masetero.

En el caso de la actividad muscular de maseteros y temporales, investigadores como Cosme et al. (2005) encontraron que la fuerza de mordida máxima ejercida durante episodios de bruxismo durante el sueño podía exceder la fuerza de apretamiento máximo voluntario, esto se explica porque durante la vigilia el reflejo protector inhibe la contracción muscular excesiva, pero durante el sueño, este sistema inhibitorio no está activo .

En esta investigación el promedio de episodios de hipopnea en los pacientes jóvenes con bruxismo del sueño fue de 28,9 . Los sujetos con BS presentan episodios de apnea e hipopnea, que se caracterizan por la disminución o bloqueo de la respiración induciendo así a un estado orgánico de hipo-oxigenación y a su vez a micro-despertares como una reacción fisiológica de protección del organismo (Del Río et al., 1997) Estos resultados son a los obtenidos por Coelho et al. (2012) quienes obtuvieron una prevalencia de 
alteraciones respiratorias como hipopneas y apneas en el $5,28 \%$ de sujetos con BS, sin asociación con la edad la edad o con el sexo $(p \leq 0.05)$. Esta condición respiratoria presente en éstos pacientes es un desafío importante en su manejo, ya que se requiere que todos los problemas clínicos asociados al BS sean evaluados por un equipo multidisciplinario.

Por causa de la apnea e hipopnea el sistema simpático que regula los órganos internos se activa y junto con la aparición de micro-despertares, se provoca un aumento en la actividad cerebral, acelerando la frecuencia cardíaca (Engleman et al., 1997). Con el incremento de la frecuencia e intensidad de los movimientos rítmicos mandibulares se podrían inducir alteraciones en el sistema estomatognatico (Del Río et al.).

Es esta investigación se observó un promedio 84,2 movimientos periódicos de las extremidades inferiores durante la duración del sueño. Esto es similar a los resultados de Bader et al. (1997) donde los sujetos con BS tuvieron significativamente más movimientos periódicos de las extremidades en comparación con los controles con una diferencia especialmente obvia para movimientos de corta duración ( $<5$ segundos). Las diferencias se hicieron significativas a partir de la cuarta hora del sueño. Según Bader et al. los sujetos con BS presentan trastornos del movimiento que incluyen apretar o rechinar los dientes y movimientos corporales de corta duración, en especial de las extremidades inferiores.

En el presente trabajo se registró un promedio de 40,60 eventos de bruxismo por hora y un número promedio de eventos de bruxismo en REM de 120,2 durante las horas de sueño. Estos resultados son similares a los obtenidos por Macaluso et al. (1998) quienes reportaron unos episodios de bruxismo distribuidos equivalentemente entre el sueño REM y no REM, con mayor promedio de eventos en las etapas 1 y $2(p<0,05)$. La mayoría de los episodios de bruxismo detectados en el sueño no $\operatorname{REM}(88 \%)$.

El conocimiento de la fisiología durante la noche en sujetos con BS y su actividad muscular durante la masticación es relevante en la odontología debido a que varias enfermedades orales degenerativas parecen estar relacionadas con una carga excesiva ejercida por la actividad musculatoria fuerte que ocurre durante el BS. Aún no hay un con- senso acerca de la cantidad y el tipo de actividad de BS para definir el límite entre lo fisiológico y lo patológico, este límite tendría definición en la respuesta individual a esta actividad.

Limitaciones del estudio. Los exámenes de polisomnografía tomados en un laboratorio del sueño, son considerados sistemas precisos, pero el estrés mental y físico de un laboratorio no debe ser desconocido.

\section{AGRADECIMIENTOS}

Los autores desean expresar su sincero agradecimiento a Mónica Naranjo y Elizabeth Macharia, quienes trabajan en el Centro de Traducción de la Universidad Autónoma de Manizales, por traducir y revisar este resumen.

LÓPEZ-S. O. P.; LÓPEZ-S. L. M.; LÓPEZ- E. Y. P.; GARIBAY-P. C. A. \& CARMONA, R. B. Oximetry, heart rate, apnea and mastication muscle activity in subjects with sleep bruxism. Int. J. Odontostomat., 14(1):42-47, 2020.

ABSTRACT: The objective of the study was to describe the average oximetry, heart rate, and apnea events values during sleep, and the activity of masseter and temporal muscles in a group of young adults with SB (Sleep Bruxism). This serial case and observational descriptive study comprised of a sample of 20 young people between 21 and 25 years of age, who were diagnosed with SB through a polysomnography test. Electromyography recordings served to describe the activity conditions of masseter and temporal muscles, while polysomnography was used to obtain measurement values and vital signs. A descriptive statistical analysis accounting for measures of central tendency, absolute and relative frequencies was performed. During mastication, the average masseter and temporal muscle activity values registered at (D: 86) (I: 520), and (D: 340) (I: 510) respectively. While, the average value for hypopnea episodes was (28.9), and (NREM: 93.5) (REM: 93.8) for oximetry. The average heart rate exhibited a value of (NREM: 65.4) (REM: 66.8) beats / minute, while the values for bruxism events registered at (NREM: 135.4) (REM: 120.2) with an index value of (SB events / hour) (40.60). Muscles of mastication characteristics, and vital signs during sleep for subjects with SB can aid in understanding and decision making in treatment.

KEY WORDS: bruxism (MeSH), sleep bruxism (MeSH), chewing (MeSH), polysomnography (MeSH), dental occlusion (MeSH), muscle contraction (MeSH). 


\section{REFERENCIAS BIBLIOGRÁFICAS}

Bader, G.; Kampe, T. \& Tagdae, T. Body movement during sleep in subjects with long-standing bruxing behavior. Int. J. Prosthodont., 13(4):327-33, 1997.

Carlsson, G. E.; Egermark, I. \& Magnusson, T. Predictors of bruxism, other oral parafunctions, and tooth wear over a 20 -year followup period. J. Orofac. Pain, 17(1):50-7, 2003.

Carra, M. C.; Rompré, P. H.; Kato, T.; Parrino, L.; Terzano, M. G.; Lavigne, G. J. \& Macaluso, G. M. Sleep bruxism and sleep arousal: an experimental challenge to assess the role of cyclic alternating pattern. J. Oral Rehabil., 38(9):635-42, 2011.

Choi, Y. S.; Choung, P. H.; Moon, H. S. \& Kim, S. G. Temporomandibular disorders in 19-year-old Korean men. J. Oral Maxillofac. Surg., 60(7):797-803, 2002.

Clark, G. T.; Beemsterboer, P. L.; Solberg, W. K. \& Rugh, J. D. Nocturnal electromyographic evaluation of myofascial pain dysfunction in patients undergoing occlusal splint therapy. J. Am. Dent. Assoc., 99(4):607-11, 1979.

Coelho, P. R.; Curcio, W. B.; do Espírito Santo, R. P.; Pereira, A. B.; Leite, F. P. P. \& Chaves, M. G. A. M. Prevalência da comorbidade entre o bruxismo do sono e a síndrome de apneia-hipopneia obstrutiva do sono: um estudo polissonográfico. Pesqui. Bras. Odontopediatria Clin. Integr., 12(4):491-6, 2012.

Cosme, D. C.; Baldisserotto, M. S.; Canabarro, S. de A. \& Shinkai, R. S. Bruxism and voluntary maximal bite force in young dentate adults. Int. J. Prosthodont., 18(4):328-32, 2005.

Del Río, S. G.; García, V. A. \& Castellanos, J. L. Apnea/hipopnea del sueño y bruxismo nocturno. Rev. A. D. M., 75(4):196-201, 1997.

Engleman, H. M.; Martin, S. E.; Deary, I. J. \& Douglas, N. J. Effect of CPAP therapy on daytime function in patients with mild sleep apnoea/hypopnoea syndrome. Thorax, 52(2):114-9, 1997.

Ferrario, V. F.; Sforza, C.; Miani, A. Jr.; D'Addona, A. \& Barbini, E. Electromyographic activity of human masticatory muscles in normal young people. Statistical evaluation of reference values for clinical applications. J. Oral Rehabil., 20(3):271-80, 1993.

Kerstein, R. B. Combining technologies: a computerized occlusal analysis system synchronized with a computerized electromyography system. Cranio, 22(2):96-109, 2004

Klasser, G. D.; Rei, N. \& Lavigne, G. J. Sleep bruxism etiology: the evolution of a changing paradigm. J. Can. Dent. Assoc., 81:f2, 2015.

Kobayashi, F. Y.; Furlan, N. F.; Barbosa, T. S.; Castelo, P. M. \& Gavião, M. B. Evaluation of masticatory performance and bite force in children with sleep bruxism. J. Oral Rehabil., 39(10):776-84, 2012.

Lavigne, G. J.; Guitard, F.; Rompré, P. H. \& Montplaisir, J. Y. Variability in sleep bruxism activity over time. J. Sleep Res., 10(3):237-44, 2001.

Lavigne, G.; Manzini, C. \& Huynh, N. T. Sleep Bruxism. In: Kryger, M. H.; Roth, T. \& Dement, W. C. (Eds.). Principles and Practice of Sleep Medicine. $5^{\text {th }}$ ed. St. Louis (Mo.), Elsevier Saunders, 2011. pp.1128-39.

Lobbezoo, F.; Ahlberg, J.; Glaros, A. G.; Kato, T.; Koyano, K.; Lavigne, G. J.; de Leeuw, R.; Manfredini, D.; Svensson, P. \& Winocur, E. Bruxism defined and graded: an international consensus. J. Oral Rehabil., 40(1):2-4, 2013.

Macaluso, G. M.; Guerra, P.; Di Giovanni, G.; Boselli, M.; Parrino, L. \& Terzano, M. G. Sleep bruxism is a disorder related to periodic arousals during sleep. J. Dent. Res., 77(4):565-73, 1998.

Manfredini, D.; Poggio, C. E. \& Lobbezoo, F. Is bruxism a risk factor for dental implants? A systematic review of the literature. Clin. Implant Dent. Relat. Res., 16(3):460-9, 2014.
Nishigawa, K.; Bando, E. \& Nakano, M. Quantitative study of bite force during sleep associated bruxism. J. Oral Rehabil., 28(5):485-91, 2001.

Onozuka, M.; Fujita, M.; Watanabe, K.; Hirano, Y.; Niwa, M.; Nishiyama, K. \& Saito, S. Mapping brain region activity during chewing: a functional magnetic resonance imaging study. J. Dent. Res., 81(11):743-6, 2002.

Raphael, K. G.; Janal, M. N.; Sirois, D. A.; Dubrovsky, B.; Wigren, P. E.; Klausner, J. J. \& Lavigne, G. J. Masticatory muscle sleep background electromyographic activity is elevated in myofascial temporomandibular disorder patients. J. Oral Rehabil., 40(12):883-91, 2013.

Raphael, K. G.; Santiago, V. \& Lobbezoo, F. Is bruxism a disorder or a behaviour? Rethinking the international consensus on defining and grading of bruxism. J. Oral Rehabil., 43(10):791-8, 2016.

Restrepo, C. C.; Alvarez, E.; Jaramillo, C.; Vélez, C. \& Valencia, I. Effects of psychological techniques on bruxism in children with primary teeth. J. Oral Rehabil., 28(4):354-60, 2001.

Saito, M.; Yamaguchi, T.; Mikami, S.; Watanabe, K.; Gotouda, A.; Okada, K.; Hishikawa, R.; Shibuya, E. \& Lavigne, G. Temporal association between sleep apnea-hypopnea and sleep bruxism events. J. Sleep Res., 23(2):196-203, 2013.

Toro, A.; Buschang, P. H.; Throckmorton, G. \& Roldán, S. Masticatory performance in children and adolescents with Class I and II malocclusions. Eur. J. Orthod., 28(2):112-9, 2006.

Trindade, M. de O. \& Rodriguez, A. G. Polysomnographic analysis of bruxism. Gen. Dent., 62(1):56-60, 2014.

Dirección para correspondencia:

Olga Patricia López Soto

Universidad Autónoma de Manizales

Antigua Estación del Ferrocarril Manizales

Manizales

COLOMBIA

Email: sonrie@autonoma.edu.co

Recibido : 06-08-2019

Aceptado: 10-09-2019 